Particle Accelerators, 1988, Vol. 23, pp. 133-148

Reprints available directly from the publisher

Photocopying permitted by license only

(C) 1988 Gordon and Breach Science Publishers, Inc.

Printed in the United States of America

\title{
TRANSVERSE COLLECTIVE INSTABILITY IN THE PSR
}

\author{
D. NEUFFER (AT-6) E. COLTON, G. SWAIN, H. THIESSEN (MP-14)
}

B. BLIND, R. HARDEKOPF, A. JASON, G. LAWRENCE, R. SHAFER

(AT-3) T. HARDEK, J. HURD, R. MACEK, M. PLUM (MP-5)

Los Alamos National Laboratory, Los Alamos, New Mexico 87545

An instability in the Proton Storage Ring (PSR) is observed as fast loss of beam during accumulation and storage when the injected beam current exceeds a threshold value. Experimental study results of that instability are presented in this paper. The instability threshold depends significantly on rf voltage, injected momentum spread, and PSR sextupole and octupole strengths. The results are consistent with the hypothesis that the instability is a transverse instability excited by a transverse impedance $Z_{\perp}$ with $\left|Z_{\perp}\right| \cong 1 \mathrm{M} \Omega / \mathrm{m}$. The driving impedance has significant magnitude in the 30 - to $300-\mathrm{MHz}$ frequency range.

\section{INTRODUCTION}

The Proton Storage Ring (PSR) is a fast-cycling high-current storage ring designed to accumulate long beam pulses $(750 \mu \mathrm{s})$ from the LAMPF linear accelerator to provide short beam pulses $(<0.36 \mu \mathrm{s})$ that drive a spallation neutron source. ${ }^{1}$ Figure 1 shows the ring, and Table I summarizes PSR parameters. The PSR is designed to provide $100-\mu \mathrm{A}$ average current at $12 \mathrm{~Hz}$, which implies accumulation of $5.2 \times 10^{13}$ protons/pulse. It was commissioned in April 1985 and has succeeded in accumulating $3.8 \times 10^{13}$ protons/pulse. Average currents of $30 \mu \mathrm{A}$ have been obtained, but beam losses inside the ring during accumulation and during extraction, as well as $\mathrm{H}^{-}$ion source problems, have so far prevented implementation of full-intensity operation.

The PSR is also useful in studies of beam dynamics. Attempts to store high-intensity beam were limited by an instability that caused beam loss on a time scale of $\sim 10$ to $100 \mu \mathrm{s}$. Figures $2 \mathrm{~A}$ and $2 \mathrm{~B}$ show stored beam as a function of time with stable and unstable conditions. The instability is dependent upon peak stored current, and the instability threshold current increases with increased rf bunching voltage.

In this paper, we summarize some of the qualitative and quantitative measurements of this instability that we have made during PSR development runs (September through December 1986). The evidence indicates that the instability is a transverse collective instability. Dependence of instability thresholds on $\mathrm{rf}$ voltage, beam momentum spread, sextupole strengths, and octupole strengths are discussed. The exciting transverse impedance is estimated at $Z_{\perp} \cong 1 \times 10^{6} \Omega / \mathrm{m}$, with $\operatorname{Re}\left(Z_{\perp}\right) \geqslant 0.25 \mathrm{M} \Omega / \mathrm{m}$, and appears to have a high-frequency spectrum $(30-300 \mathrm{MHz})$. The source of the impedance has not yet been identified. Some possibilities are suggested and will be the subject of further studies. 


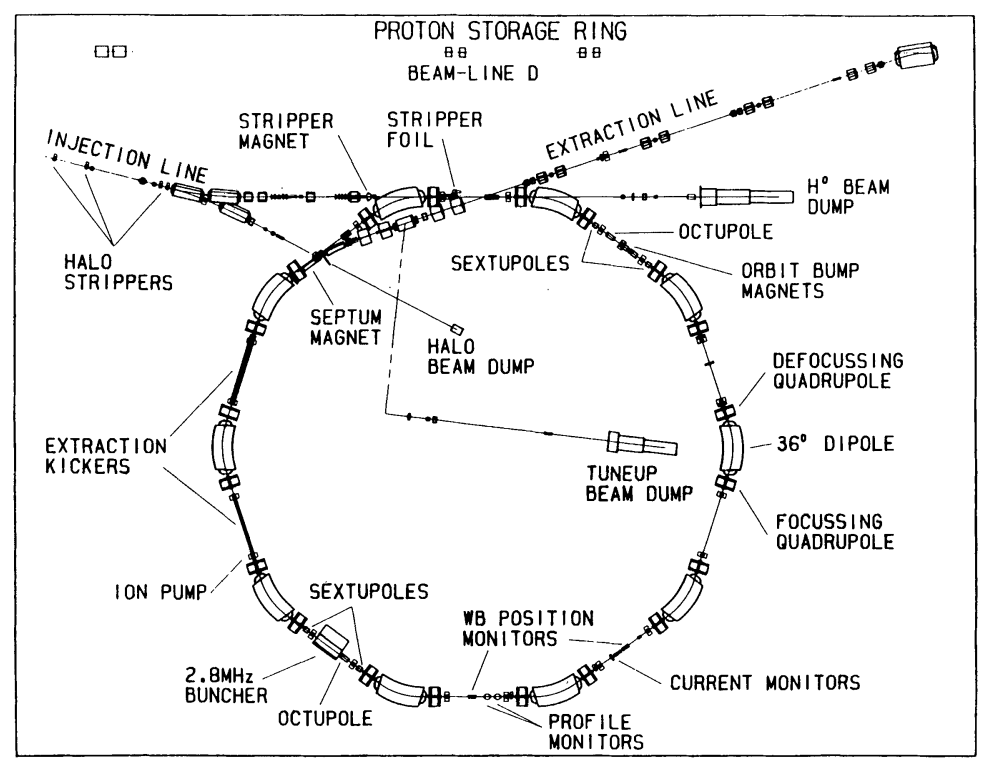

FIGURE 1 The Proton Storage Ring (PSR).

\section{TRANSVERSE AND LONGITUDINAL INSTABILITIES IN THE PSR}

There have been many studies on the possible effects of transverse and longitudinal instabilities. ${ }^{2-4}$ The discussions are simplest in the case of "coasting" or "unbunched" beam, and we follow Refs. 3 and 4 to find expressions for the stability thresholds and the growth rates of possible instabilities. The PSR operates with a harmonic $h=1 \mathrm{rf}$ system so that it contains only one bunch, and multibunch instabilities cannot appear. As discussed below, the wavelength of the unstable motion is much less than the bunch length, and the instability growth

TABLE I

PSR Parameters

\begin{tabular}{lll}
\hline Proton kinetic energy & $T$ & $797 \mathrm{MeV}$ \\
Circumference & $2 \pi R$ & $90.2 \mathrm{~m}$ \\
Average beam-pipe radius & $b$ & $0.05 \mathrm{~m}$ \\
Betatron tunes & $v_{x}, v_{y}$ & $3.2,2.2$ \\
Transition gamma & $\gamma_{\mathrm{T}}$ & 3.1 \\
Betatron amplitudes & $\beta_{\min }, \beta_{\max }$ & $1.5 \mathrm{~m}, 17.0 \mathrm{~m}$ \\
Dispersion & $\eta_{\min }, \eta_{\max }$ & $1.1 \mathrm{~m}, 2.5 \mathrm{~m}$ \\
Chromaticities (measured) & $\xi_{x}, \xi_{y}$ & $-1.3,-1.0$ \\
Buncher harmonic, frequency & $h, f$ & $1,2.795 \mathrm{MHz}$ \\
Peak rf voltage & $V$ & $15 \mathrm{kV}$ \\
Maximum synchrotron tune & $v_{\mathrm{s}}$ & 0.0006 \\
Design stored beam & $N_{\max }$ & $5.2 \times 10^{13}$ \\
Design peak current & $I_{\max }=2 \bar{I}$ & $46.3 \mathrm{~A}$ \\
\hline
\end{tabular}



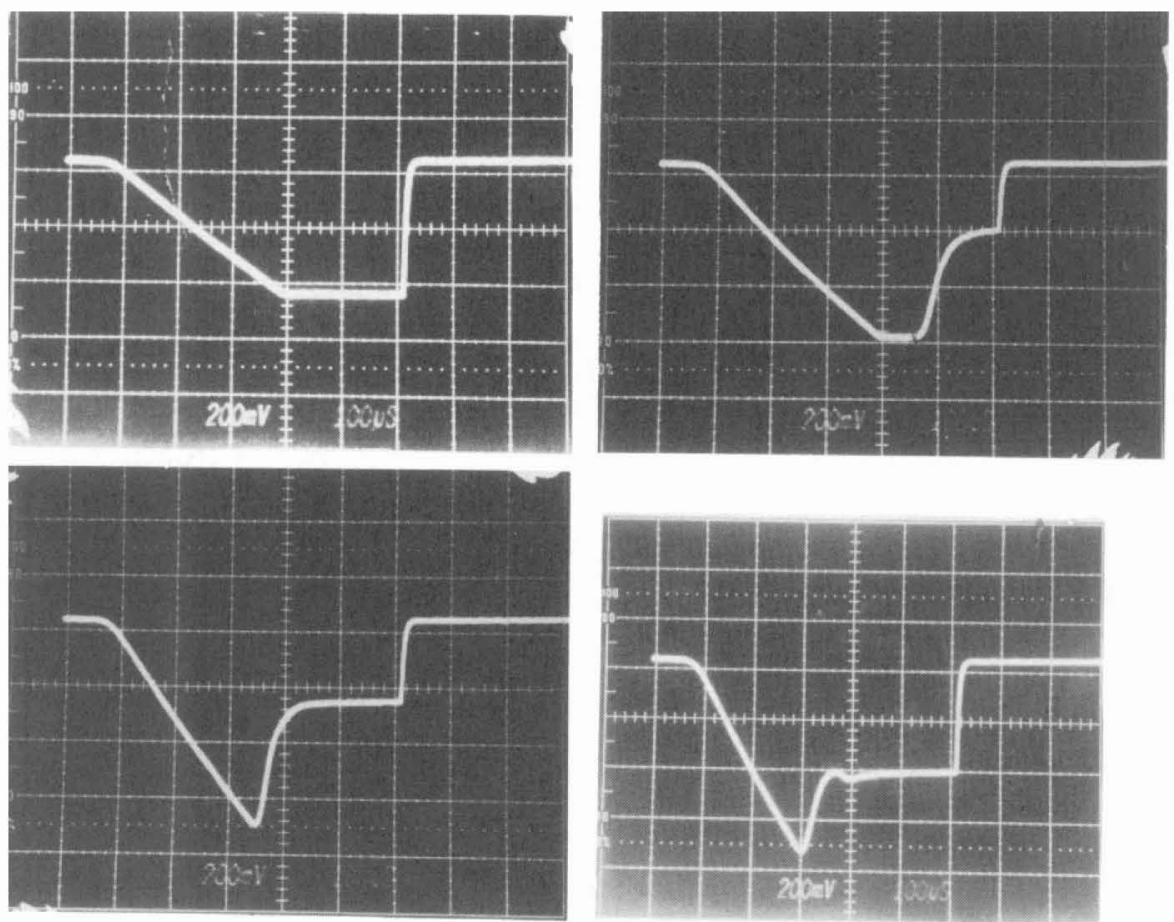

FIGURE 2 Appearance of the instability. A: Beam current as a function of time in a stable beam pulse with extraction $500 \mu$ s after injection. The stored-beam intensity is $\sim 6 \times 10^{12}$ protons ( $\mathrm{rf}$ is off). B: With higher injected current, the beam becomes unstable with fast beam loss $\sim 100 \mu \mathrm{s}$ after injection. C: With still larger injected current, the fast beam loss occurs before injection is completed. D: With larger injected current, the fast beam loss occurs earlier in injection.

time is much less than a typical PSR synchrotron period. Therefore, the PSR bunched-beam instability thresholds would be the same as those for a coasting beam, with peak currents replacing the average coasting-beam current in the threshold expressions. Most of the experiments discussed below were, in fact, performed with unbunched beam (rf off) to provide the simplest conditions for study of the instability.

The transverse instability limit for coasting beam can be written as

$$
\left|I Z_{\perp}\right| \approx\left|\frac{4 F_{\perp} E_{0} \beta \gamma}{e \beta_{\perp}}\right| \Delta v,
$$

where $I$ is the beam current, $Z_{\perp}$ is the transverse impedance, $F_{\perp}$ is a factor of order unity that depends on the bunch distribution and the impedance phase, $E_{0}$ is the proton rest energy, $\beta_{\perp}$ is the average transverse betatron amplitude, and $\Delta v$ is the total tune spread of the beam. If there are no octupoles or higher-order nonlinearities,

$$
\Delta v=|(n-v) \eta-\xi v| \frac{\Delta p}{p},
$$


where $n$ is the harmonic number of the impedance, $v$ is the betatron tune,

$$
\eta=\frac{1}{\gamma^{2}}-\frac{1}{\gamma_{t}^{2}}=0.19
$$

for the PSR, and $\xi$ is the chromaticity. The full momentum spread is $\Delta p / p$. Octupoles and other nonlinearities add an amplitude dependence to the tune, which adds an additional term $\Delta v_{\mathrm{NL}}$ to the tune spread.

Near instability, the growth rate may be written as

$$
\tau^{-1}=x_{1}\left(2 \pi f_{0}\right) \Delta v,
$$

where $f_{0}$ is the revolution frequency, and $x_{1}$ is a factor that approaches 0 at the instability threshold and increases to a factor of order unity at twice the threshold current. For currents greatly above threshold, there is no Landau damping, and the growth rate is given by

$$
\tau^{-1} \cong \frac{f_{0} e I \beta_{\perp} \operatorname{Re}\left(Z_{\perp}\right)}{2 \beta \gamma E_{0}} .
$$

In the PSR near the instability threshold, $\Delta v \cong 0.01$, so that

$$
\tau^{-1} \cong x_{1}(5.7 \mu \mathrm{s})^{-1} \text {. }
$$

Longitudinal coasting-beam instabilities can also occur in the PSR. The longitudinal stability criterion can be written as

$$
I\left|\frac{Z_{\|}}{n}\right| \approx F c p \beta \eta\left(\frac{\Delta p}{p}\right)^{2},
$$

where $F$ is a factor of order unity, $\beta=v / c$, and $Z_{\|} / n$ is the longitudinal impedance per harmonic number $n$. For a resistive wall, the longitudinal and transverse impedances are related by

$$
Z_{\perp}=\frac{2 R}{b^{2}} \frac{Z_{\|}}{n},
$$

and this relationship is a useful guide in comparing longitudinal and transverse impedances.

The growth rate for longitudinal instabilities may be expressed as

$$
\tau^{-1}=n \pi f_{0} \eta x_{2} \frac{\Delta p}{p},
$$

where $x_{2}$ is a factor that becomes zero at the instability threshold and is of order 0.3 at a current of twice the threshold. At PSR parameters $\left(\Delta p / p=2 \times 10^{-3}\right.$, $\eta=0.19$ ),

$$
\tau^{-1} \cong n x_{2}\left(300 \mu \mathrm{s}^{-1}\right) .
$$

In the highly unstable limit without Landau damping,

$$
\tau^{-1} \cong n f_{0} \operatorname{Im}\left[\left(\frac{i e 2 \pi \eta I Z_{\|}}{\beta^{2} \gamma E_{0} n}\right)^{\frac{1}{2}}\right] .
$$


TABLE II

Instability Limits at Sample PSR Parameters

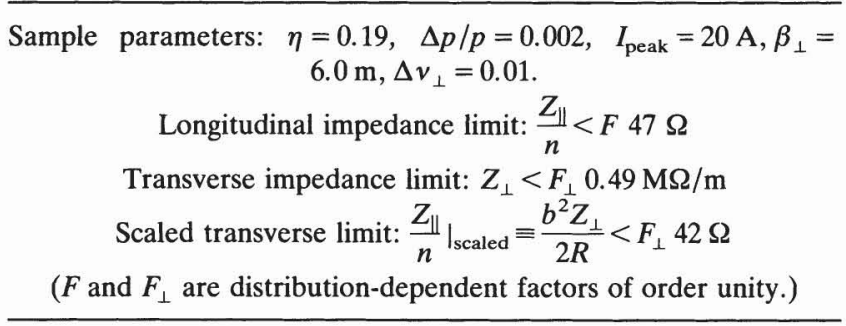

In Table II we display the impedances corresponding to the stability limits with parameters set at reference values near PSR operating conditions. The impedance limits are relatively high. The threshold for transverse instability is similar to that for longitudinal instabilities; both types of instabilities may occur in the PSR. The instability growth rates are similar for $n \cong 50$, with transverse instabilities faster at lower frequencies and longitudinal instabilities faster at high frequencies.

\section{OVERVIEW OF THE INSTABILITY}

The instability became evident through beam loss during beam accumulation or beam storage in the PSR. Figure 2 shows the instability. At low currents, the beam is stable (Fig. 2A). As more beam is stored, an instability appears that causes beam loss (Figs. 2B and 2C). As still more beam is injected, the beam becomes unstable before injection is completed (Fig. 2D).

Instability occurs when the injected beam current is above some threshold value. The instability growth rate, as estimated from the beam loss rate, is typically much faster than the synchrotron oscillation frequency. The instability threshold substantially increases, and the growth rate decreases, when the PSR buncher rf voltage increases. Transverse oscillations are noticable shortly before beam loss and continue during beam loss (see Fig. 3). Betatron sidebands of the

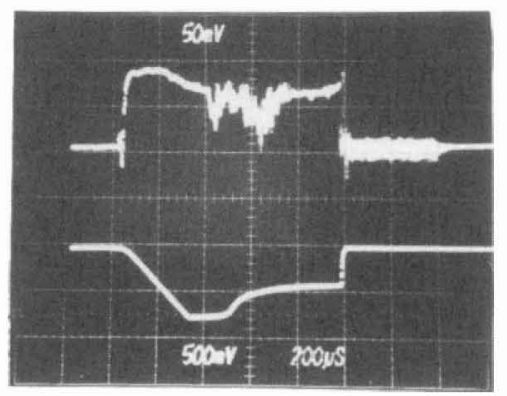

FIGURE 3 The lower sweep shows beam current as a function of time with instability. The upper sweep shows a difference signal from a transverse pickup. Transverse oscillation signals appear before the beam loss and continue until beam loss has reduced current to a stable level. 
revolution harmonics are noticeable during unstable beam pulses but are not readily seen during stable beam pulses. High-frequency signals are observable at $30-40 \mathrm{MHz}$ on the transverse beam monitors; these signals may be associated with the instability.

The instability does not greatly depend upon the vacuum conditions; increasing residual gas pressure by two orders of magnitude from $10^{-8}$ torr shows no significant change in the instability threshold current, although fast beam loss appears somewhat earlier in the beam pulse at the higher pressures. The instability also does not depend appreciably on injection stripper thickness and location. There is also no strong dependence of the instability on the vertical or horizontal tunes of the PSR.

The instability may be significant in limiting beam current in high-intensity operation with $\mathrm{rf}$ at full voltage, even with extraction immediately following injection. Figure 4A shows a beam pulse during highest-intensity operation $\left(\sim 3.2 \times 10^{13}\right.$ protons/pulse $)$. With $\mathrm{rf}$ switched off, the beam is violently unstable (Fig. 4B). With rf on, the beam is still somewhat unstable, as can be seen by beam losses in beam storage when extraction is delayed (Fig. 4C). Higherintensity operation will be difficult unless the instability is reduced.
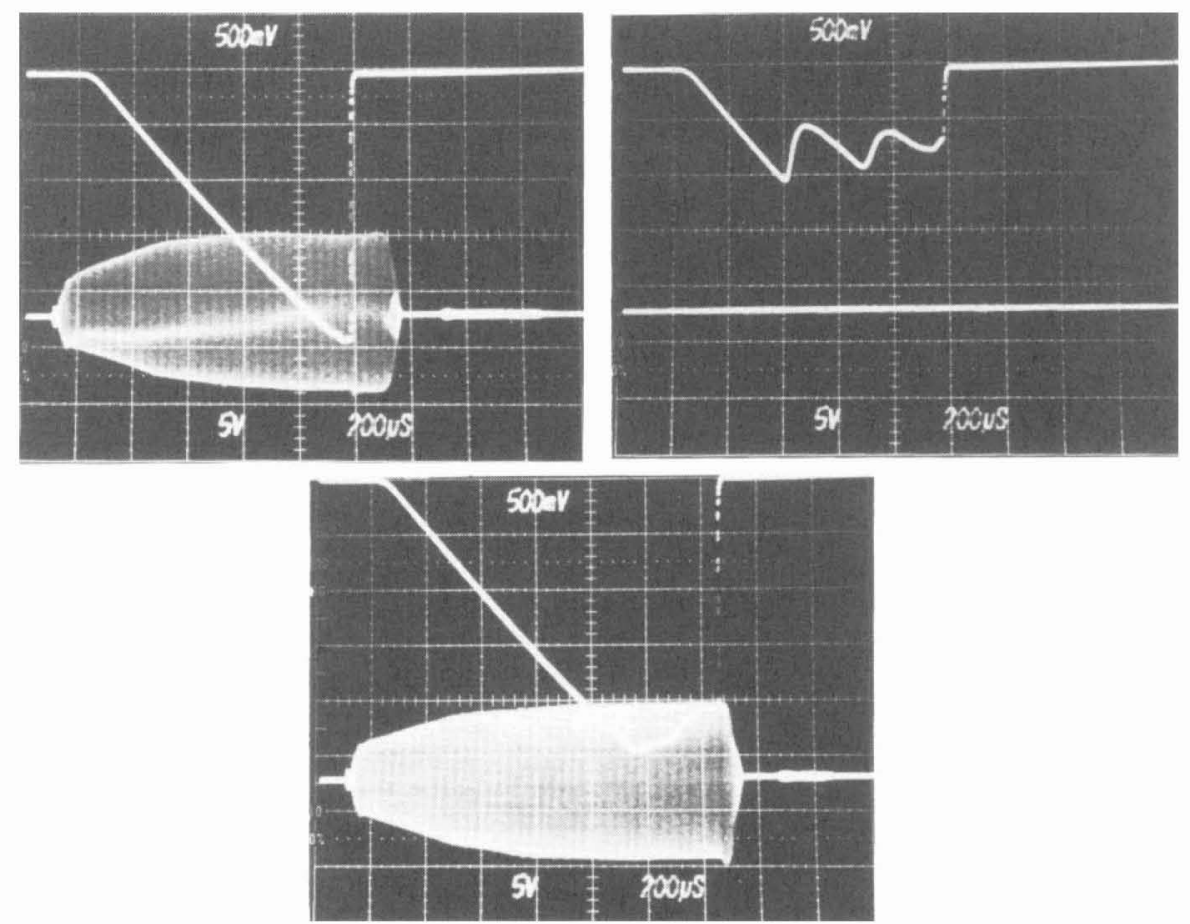

FIGURE 4 Instability in highest intensity operation. A: A beam pulse during highest operation $\left(\sim 3.2 \times 10^{13}\right.$ stored protons) with extraction immediately following injection and $\mathrm{rf}$ at maximum voltage $(14 \mathrm{kV})$. B: Same injected beam but with $\mathrm{rf}$ off. Beam is unstable with multiple beam loss events during injection. C: Same injected beam with rf on but with beam stored for $200 \mu$ s after injection. Instability appears as fast loss during storage. 
The dependence of instability threshold on rf voltage is described in more detail below, where it is correlated with the change in beam momentum spread. The momentum spread can also be changed by modifying the injected beam, resulting in significant changes in instability thresholds. The thresholds can be greatly changed by varying the PSR sextupole strengths. There is also a significant dependence on ring octupole strengths. All these instability features are described in more detail below.

\section{DEPENDENCE OF THE INSTABILITY ON RF VOLTAGE}

The PSR has a harmonic $h=1$ tunable bunching cavity $(2.8 \mathrm{MHz})$, with a maximum accelerating voltage of $\sim 15 \mathrm{kV}$. The cavity is normally on at near maximum voltage $(>10 \mathrm{kV})$ during PSR operation, and the voltage waveform (voltage as a function of time during the beam pulse) is tunable and is varied to minimize beam loss. The synchrotron period is relatively long $(600 \mu \mathrm{s}$ at $V=15 \mathrm{kV}$ ), and the instability growth times at currents significantly above threshold are usually much shorter. The instability threshold is significantly dependent on rf voltage, and that dependence was measured in a series of experiments. The results are displayed graphically in Fig. 5. In these experiments, the beam was considered stable if the beam current remained stored for $500 \mu \mathrm{s}$ after injection without any large, fast losses. Injected beam was increased until instability was reached and the maximum, stable, stored beam was recorded. The rf voltage was held constant at the reference value throughout the beam pulse.

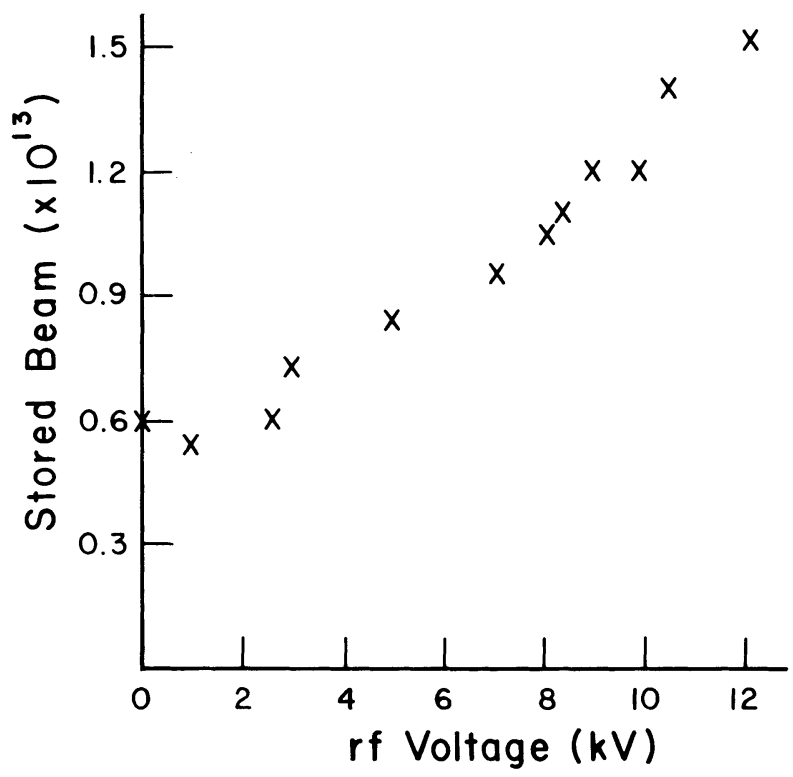

FIGURE 5 Dependence of maximum stable beam upon buncher voltage. Beam is considered stable if it is stored for $500 \mu \mathrm{s}$ after injection without fast beam loss in this set of experiments. 
The maximum stable current increases from $\sim 0.55 \times 10^{13}$ protons with $0 \mathrm{rf}$ voltage to $\sim 1.5 \times 10^{13}$ protons with $12-\mathrm{kV}$ voltage, and the increase appears to be nearly linear with voltage. If the instability is longitudinal or transverse, the threshold should change with rf voltage as the momentum spread and phase width of the beam bunch change, with the thresholds given by Eqs. (1) and (4). As the rf voltage $V$ increases, the maximum stable momentum deviation $\delta p / p$ (height of rf bucket) should increase as $V^{1 / 2}$. If injection were perfectly matched without phase-space dilution, the phase-space area of the beam $(\Delta \phi \Delta p / p)$ would be constant and $\Delta p / p$ would increase as $V^{1 / 4}$, where $\Delta \phi$ is the phase length of the beam. From Eqs. (1) and (4), it can be shown that the maximum stable current limited by transverse instability should vary as $(\Delta \phi \Delta p / p)$, and the maximum current limited by longitudinal instability should vary as $\left(\Delta \phi(\Delta p / p)^{2}\right)$.

Injection into the PSR is not matched in phase space, and considerable phase-space dilution does occur. This dilution can be estimated by a numerical simulation of PSR injection. In the simulation, beam is injected with an initial total phase spread of $\pm 2 \pi / 3$ and an initial momentum spread of \pm 0.0015 and tracked turn by turn with applied rf forces. The resulting phase and momentum spreads are displayed in Table III and graphed in Fig. 6. Figure 6 may be compared with Fig. 5. Although the increase in current threshold does follow the simulated increase in phase space reasonably well, the comparison does not provide conclusive evidence on whether the instability thresholds are transverse or longitudinal. The threshold measurements and the simulation with its assumptions contain sufficient uncertainties to make the comparison indefinite.

Momentum spread can also be changed by varying the injected beam momentum; in some experiments, the momentum spread was deliberately increased by modulating the phase of the last accelerating module of the LAMPF linac before PSR injection. In these experiments the rf was off $(V=0)$, so that the momentum width was determined by the injected beam width, and the width was varied by changing the amplitude of the phase modulation. Figure 7 displays

TABLE III

Results of Simulated Injection into the PSR ${ }^{\mathrm{a}}$

\begin{tabular}{|c|c|c|c|c|}
\hline $\mathrm{V}_{\mathrm{rf}}(\mathrm{kV})$ & $\delta \phi_{\mathrm{rms}}$ & $\begin{array}{c}\Delta p / p_{\text {full }} \\
\left(2.35 \delta p / p_{\text {rms }}\right)(\%)\end{array}$ & $\delta \phi \Delta p / p(\%)$ & $\delta \phi(\Delta p / p)^{2}(\%)$ \\
\hline 0.0 & 1.56 & $0.22 \%$ & $0.34 \%$ & $0.076 \%$ \\
\hline 2.5 & 1.256 & 0.29 & 0.307 & 0.089 \\
\hline 5.0 & 1.250 & 0.37 & 0.37 & 0.137 \\
\hline 7.5 & 0.97 & 0.44 & 0.43 & 0.188 \\
\hline 10.0 & 0.92 & 0.52 & 0.48 & 0.25 \\
\hline 12.5 & 0.91 & 0.58 & 0.53 & 0.306 \\
\hline 15.0 & 0.91 & 0.625 & 0.57 & 0.355 \\
\hline
\end{tabular}

${ }^{a}$ In the simulations, an initial momentum spread of 0.003 and an initial full-phase spread of $250^{\circ}$ were assumed. Energy loss in the foil was not included. 


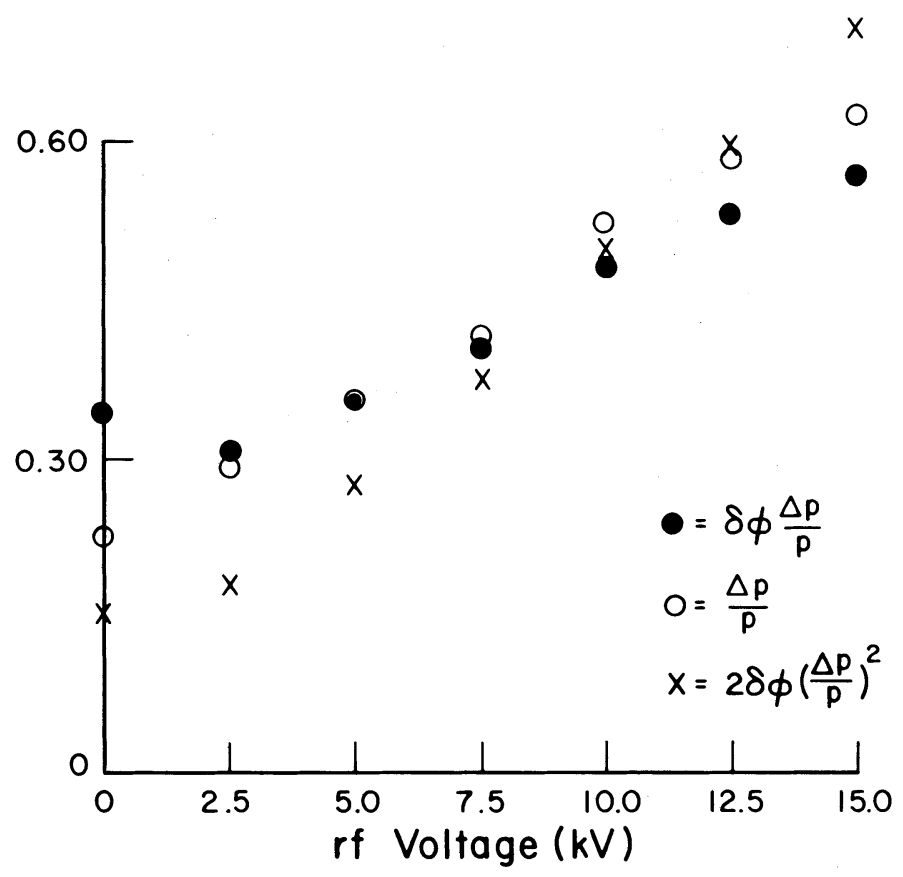

FIGURE 6 Dependence of momentum spread and phase spread of stored beam upon rf voltage-simulation results. Stable beam current should increase as $(\Delta \varphi \Delta p / p)$ with transverse instability and as $\left(\Delta \varphi(\Delta p / p)^{2}\right)$ with longitudinal instability. Both dependences are shown.

some results of the experiments. The results show an increase in maximum stable beam from $6 \times 10^{12}$ with $\Delta p / p \cong 0.002$ (no modulation) to $1.8 \times 10^{13}$ at maximum modulation $(\Delta p / p \cong 0.008)$, and the results are consistent with a linear increase of threshold with $\Delta p / p$, as would be expected with a transverse instability, although there are significant uncertainties in the results.

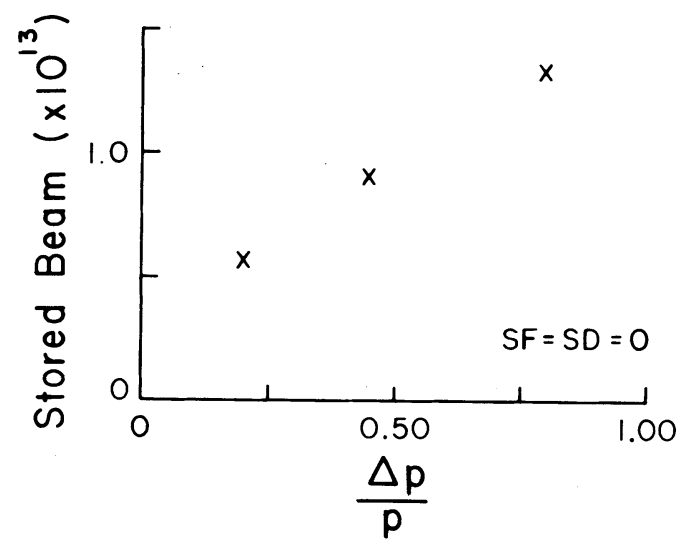

FIGURE 7 Dependence of maximum stored beam on $\Delta p / p$. Beam was considered stable if stored for $500 \mu \mathrm{s}$ without fast beam loss. Momentum spread was varied by varying injected momentum spread. 


\section{EXPERIMENTS WITH SEXTUPOLES}

Two independent sets of sextupoles (two pairs) were installed in the PSR in November 1986. The sextupoles are located in two straight sections on opposite sides of the PSR with one set close to focusing quads where the lattice functions are $\beta_{x} \cong 10.0 \mathrm{~m}, \beta_{y} \cong 3.0 \mathrm{~m}$, and $\eta \cong 2.3 \mathrm{~m}$ (the $F$ sextupoles); the other set is close to defocusing quads where $\beta_{x} \cong 2.0 \mathrm{~m}, \beta_{y} \cong 12.0 \mathrm{~m}$, and $\eta \cong 1.3 \mathrm{~m}$ (the $D$ sextupoles). Locating the sextupoles on opposite sides of the ring cancels the excitation of odd resonances. The lowest-order dynamic effects of the sextupoles are changes in the $x$ and $y$ chromaticities of the ring. These changes may be calculated from the expressions

$$
v_{x} \Delta \xi_{x}=\sum_{i} \frac{\eta \beta_{x}}{2 \pi B \rho} S_{i} L_{i}
$$

and

$$
v_{y} \Delta \xi_{y}=-\sum_{i} \frac{\eta \beta_{y}}{2 \pi B \rho} S_{i} L_{i},
$$

where the sum is taken over all sextupoles. The length of a sextupole is $L_{i}$, $S_{i}=B_{i}^{\prime \prime} / 2=B_{i} / a^{2}$ is the field strength of a sextupole, and the lattice parameters $\beta$, $\eta$ are evaluated at the sextupole locations. The two independent sets of sextupoles permit independent variation of $x$ and $y$ chromaticities. The length of a PSR sextupole is $0.3 \mathrm{~m}$, and its maximum strength is $24 \mathrm{~T} / \mathrm{m}^{2}$ with a current $I=20 \mathrm{~A}$. The expected chromaticities of the PSR as a function of the $D$ and $F$ sextupole currents are

$$
\xi_{x}=-0.82+0.17 I_{F}-0.019 I_{D}
$$

and

$$
\xi_{y}=-1.3-0.074 I_{F}+0.17 I_{D} \text {, }
$$

where $I_{\mathrm{F}}$ and $I_{\mathrm{D}}$ are in A and their signs have been selected so that positive values yield positive $x$ and $y$ chromaticities. The calculated values at zero sextupole strength are found assuming an idealized lattice with rectangular dipoles and no other nonlinearities. Experiments have determined the following expressions for the PSR chromaticity:

$$
\xi_{x}=-1.37+0.16 I_{\mathrm{F}}-0.015 I_{\mathrm{D}}
$$

and

$$
\xi_{y}=-1.10-0.066 I_{\mathrm{F}}+0.16 I_{\mathrm{D}} .
$$

The effects of the sextupole fields agree quite well with the calculated values. The differences between calculated and measured chromaticities at $I_{\mathrm{F}}=I_{\mathrm{D}}=0$ are probably caused by nonlinearities in the real PSR that are not included in the idealized lattice.

At the PSR, a series of experiments was performed that explored the dependence of the instability on sextupole strengths. Stable beam current was 


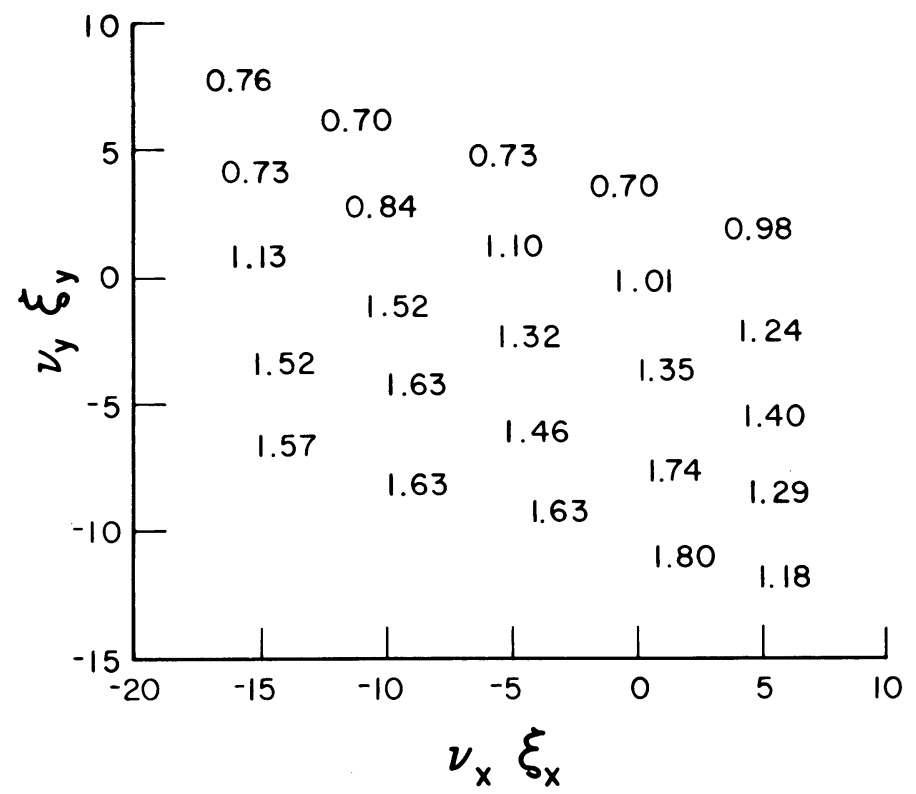

FIGURE 8 Stable current as function of $v_{x} \xi_{x}$ and $v_{y} \xi_{y}$. In these experiments rf was off, momentum spread was set at $\sim 0.008$, and beam was considered stable if stored for $500 \mu \mathrm{s}$ without fast beam loss.

indeed strongly dependent on the magnitude and sign of the chromaticity. Figure 8 shows maximum stored beam without instability as a function of $\xi_{x}$ and $\xi_{y}$ for a set of experiments in which injected beam had a large momentum spread $(\Delta p / p \cong 0.008)$ and $\mathrm{rf}$ voltage was off. Stable current varies from $7 \times 10^{12}$ to $1.8 \times 10^{13}$ protons, depending on sextupole strengths. Similar experiments were also performed with different initial momentum spreads and with rf on.

The results of these experiments may be summarized as follows:

1. The highest stable currents were found at large negative vertical chromaticity $\left(\xi_{y} \cong-4\right)$, with horizontal chromaticity simultaneously not greatly positive $\left(\xi_{x}<0.5\right)$.

2. The instability was more dependent on vertical than on horizontal chromaticity; however, it was not independent of $\xi_{x}$, as noted above.

3. The dependence on chromaticity was greatly reduced with a smaller momentum spread. The reduction is consistent with a linear dependence on $\Delta p / p$; however, as discussed above, that dependence is not conclusively determined.

The results support the hypothesis that the instability is transverse rather than longitudinal because a transverse instability is expected to depend on chromaticity, whereas a longitudinal one does not. The results may be correlated with the tune-spread expression

$$
\Delta v \cong|[(n-v) \eta-\xi v]| \frac{\Delta p}{p}+\Delta v_{\mathrm{NL}}
$$


with the following comments:

1. The greater dependence on vertical chromaticity does not imply that the vertical impedance is much greater than the horizontal because the stronger horizontal focusing makes the beam a factor of $\left(v_{x} / v_{y}\right)$ more sensitive to vertical impedances. Because the instability is not independent of $\xi_{x}$, perhaps the horizontal impedance is in fact not greatly less than the vertical.

2. The fact that a region of somewhat improved stability was not found at largest positive chromaticities suggests that the instability is concentrated at relatively high harmonics $(n>10)$ or that it covers a broad range of harmonics. The low harmonics $(n \cong v)$ are not dominant.

3 . The fact that the instability is strongly $\xi$ dependent implies that the instability must be concentrated at harmonics that are not too high $(n<100)$.

\section{DEPENDENCE ON OCTUPOLE STRENGTH}

Two octupoles were inserted at opposite sides of the PSR in December 1986. They were placed in the center of the straight sections, where $\beta_{x} \cong \beta_{y}(6 \mathrm{~m})$ and $\eta \cong 2 \mathrm{~m}$. The lowest-order effect of the octupoles is to give particles in the beam amplitude- and momentum-dependent tune shifts and, therefore, give the beam a nonlinear tune spread. The tune shifts may be found from

$$
\Delta v_{x}=\sum \frac{B^{\prime \prime \prime} L_{0} \beta_{x}}{32 \pi B \rho}\left[A_{x}^{2}-2 A_{y}^{2}+4 \eta\left(\frac{\delta p}{p}\right)^{2}\right]
$$

and

$$
\Delta v_{y}=\sum \frac{B^{\prime \prime \prime} L_{0} \beta_{y}}{32 \pi B \rho}\left[A_{y}^{2}-2 A_{x}^{2}-4 \eta\left(\frac{\delta p}{p}\right)^{2}\right],
$$

where the sum is over all octupoles of lengths $L_{0}$ and strengths $B^{\prime \prime \prime}=6 B / a^{3}$, the lattice functions are to be evaluated at the octupoles, and $A_{x}=\sqrt{\beta_{x} \varepsilon_{x}}$ and $A_{y}$ are the particle amplitudes at the octupoles. For the PSR, the octupole length is $0.44 \mathrm{~m}$ and the octupole strength is $B^{\prime \prime \prime}=4580 \mathrm{~T} / \mathrm{m}^{3}$ at 200 -A current. For typical PSR parameters $\left(\varepsilon_{0}=20 \times 10^{-6}, \delta p / p=\delta \times 10^{-3}\right)$, we obtain

$$
\Delta v_{x}=0.006\left[\frac{\varepsilon_{x}}{\varepsilon_{0}}-2 \frac{\varepsilon_{y}}{\varepsilon_{0}}\right]+0.067 \delta^{2} .
$$

The PSR showed a significant dependence of stable beam upon octupole current. Figure 9 shows results of an experiment in which octupole strength was varied. The rf was off in that experiment, and the injected momentum spread was not enhanced. Stable beam increased from $6 \times 10^{12}$ at zero octupole current to $1.5 \times 10^{13}$ at $225 \mathrm{~A}$. The stable beam was injection-current-limited at higher fields.

The results support the hypothesis that the instability is transverse rather than longitudinal because a longitudinal instability would have no dependence on 


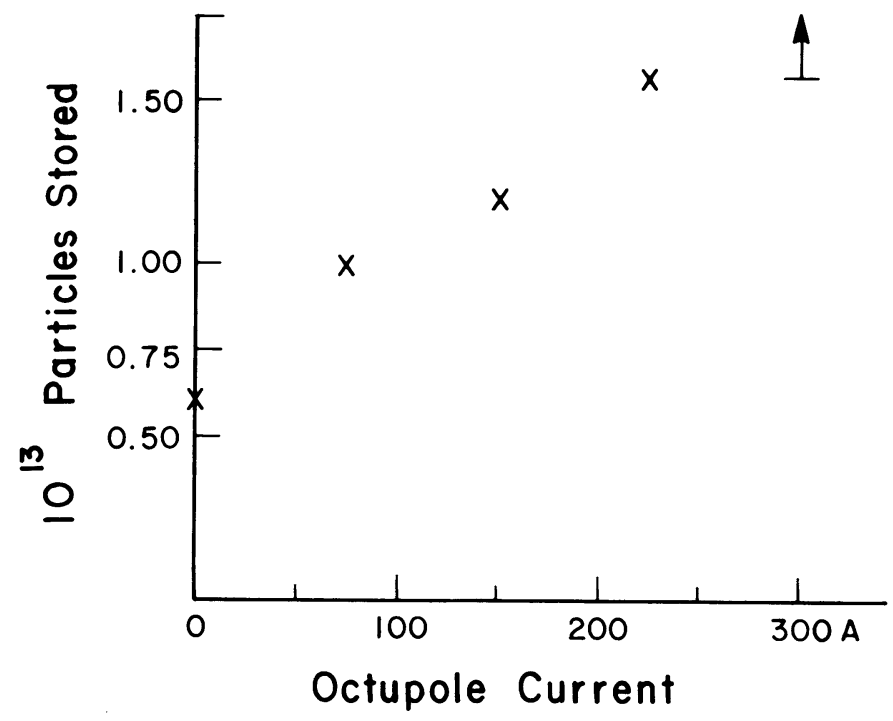

FIGURE 9 Stable stored beam as a function of current in PSR octupoles. In these experiments $\mathrm{rf}$ was off, $\Delta p / p \cong 0.002$ was not enhanced, and beam was considered stable if stored for $100 \mu$ s after the end of injection without fast beam loss. Intensity was injection limited at $\sim 1.5 \times 10^{13}$.

octupoles, whereas a transverse one can be significantly damped by octupoleinduced tune spreads. The data (Fig. 9) may be fitted by a model of the form

$$
\Delta v=\Delta v_{0}+\frac{d(\Delta v)}{d I_{0}} I_{0}
$$

where $I_{0}$ is the magnitude of the octupole current and $\Delta v \cong 0.01$ contains the bare-machine nonlinearities plus the tune spread due to the injected momentum spread. In the experiments, there was also a weak dependence of the stability on the sign of the octupoles, indicating some interference between octupole and space charge effects, according to Möhl and Schönauer, ${ }^{5}$ or possibly some interference with other PSR nonlinearities.

\section{SOURCES OF THE IMPEDANCE}

The experimental results are consistent with a transverse instability driven by an impedance with a magnitude of $\sim 1.0 \mathrm{M} \Omega / \mathrm{m}$. Inserting best estimates for the PSR parameters into the transverse instability threshold expression [Eq. (1)], we obtain $\left|Z_{\perp}\right| \cong 0.7 F_{\perp} \mathrm{M} \Omega / \mathrm{m}$ for the driving impedance. The impedance should also have high-frequency content $(30-300 \mathrm{MHz})$. The exact source of this impedance has not yet been identified. In this section we suggest some possibilities. A more detailed discussion of the known devices in the PSR and their estimated impedances has been presented in Ref. 6 , and some of the results are included in the present discussion. 
Contributions to the impedance will be obtained from all components of the ring, which include the beam-pipe resistance, rf cavity resonances, bellows, steps in beam-pipe diameter, bump magnets, kicker plates, beam monitors, the stripper-foil unit, capacitive pickups, and beamline junctions. Some of the more significant contributions are expected from the following.

1. Space-charge impedance. The PSR has a relatively large space-charge impedance given by

$$
Z_{\perp}=i \frac{R Z_{0}}{\beta^{2} \gamma^{2}}\left[\frac{1}{a^{2}}-\frac{1}{b^{2}}\right],
$$

where $Z_{0}$ is the impedance of free space, $a$ is the beam radius, and $b$ is the beam-pipe radius. At PSR parameters, the resulting impedance is $Z_{\perp} \cong$ $i 10 \mathrm{M} \Omega / \mathrm{m}$. However, this impedance is purely imaginary and does not directly drive the instability. For typical dispersion relations, however, the threshold is lowered for $\operatorname{Re}\left(Z_{\perp}\right)$ at which instability is seen. From the instability growth rates, we estimate that $\operatorname{Re}\left(Z_{\perp}\right) \simeq 0.25 \mathrm{M} \Omega / \mathrm{m}$ is needed.

2. Resistive-wall impedance. The resistive wall from the PSR beam pipe (5-cm radius stainless steel) is only expected to contribute less than $10^{4} \Omega / \mathrm{m}$ in the 30 to $300-\mathrm{MHz}$ frequency region.

3. Fast kickers. The PSR contains two fast kickers of 3.95-m length. Assuming that the kickers can be treated as strip lines with a $50-\Omega$ characteristic impedance, $Z_{\perp} \cong 0.1 \mathrm{M} \Omega / \mathrm{m}$ is obtained.

4. Bump magnets. The bump magnets are simple cylindrical cavities with a single-turn coil. The coil may be approximated as a transmission line with characteristic impedance of $700 \Omega$, with a resulting total impedance of $\sim 0.3 \mathrm{M} \Omega / \mathrm{m}$ at $\sim 100 \mathrm{MHz}$ from the six bump magnets. There also will be cavity modes in the impedance.

5. Buncher cavity. Measurements indicate that, at the fundamental frequency, the buncher cavity is a simple resonator with a shunt impedance less than $70 \Omega$ and a very low $Q$. A few parasitic modes were also seen in the 1 - to $1000-\mathrm{MHz}$ range, but the measured impedances were small.

6. Other structures. The various irregularities in the beam pipe-cross-section changes, ports, bellows, etc.-are believed to sum into a wideband impedance with $Q \cong 1$ of undetermined magnitude, which may or may not be sufficient to drive the instability.

Figure 10 shows the real and imaginary parts of the transverse impedances, calculated with the code $\mathrm{ZLT}^{6}$ using known expressions for the impedances of various devices. The calculations do not uniquely identify the source of the impedance driving the stability. Future development studies will remove the bump magnets, short circuit the buncher cavity, and modify other devices in the ring. These studies may result in a more precise identification. Also, future studies will more accurately measure the frequency structure and time dependence of the unstable motion. 

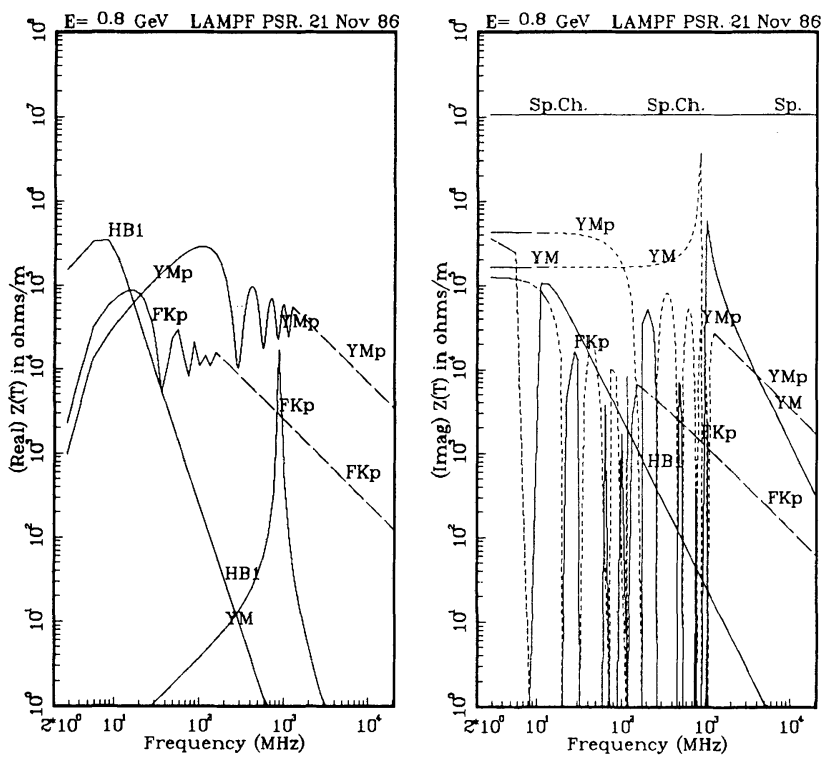

FIGURE 10 Calculated real and imaginary parts of the transverse coupling impedances for space charge in an ideal beam pipe (Sp.Ch.), fast kicker plates (FKp), buncher fundamental with rf on (HB1), the bump magnet considered as a parallel-plate transmission line (YMp), and a postulated high-frequency cavity resonance of the bump-magnet chamber (YM). (Impedance scale shown is 1 to $10^{8} \Omega / \mathrm{m}$; frequency scale is $2 \mathrm{MHz}$ to $20 \mathrm{GHz}$.)

\section{SUMMARY}

Evidence for a transverse instability has been observed at the PSR. The evidence from studies of the dependences of stable current on rf voltage, injected momentum spread, sextupole strengths, and octupole strengths is consistent with a transverse instability driven by an impedance $Z_{\perp}$ with a magnitude of $\sim 1 \mathrm{M} \Omega / \mathrm{m}$, with $\operatorname{Re} Z_{\perp} \geqslant 0.25 \mathrm{M} \Omega / \mathrm{m}$, that excites the beam at high harmonics $(n=10-100)$. The precise source of the driving impedance has not yet been identified.

\section{ACKNOWLEDGEMENT}

We are grateful for the help of R. Johnson of MP-11 and the PSR operating crew.

\section{REFERENCES}

1. G. P. Lawrence, R. A. Hardekopf, A. J. Jason, P. N. Clout, and G. A. Sawyer, IEEE Trans. Nucl. Sci. NS-32, 2662 (1985). 
2. A. Hoffman, B. Zotter, and F. Sacherer, in Theoretical Aspects of the Behaviour of Beams in Accelerators and Storage Rings, M. H. Blewett, ed., CERN report 77-13, 139-197 (1977).

3. E. Keil and W. Schnell, CERN report ISR-TH-RF/69-48 (1969).

4. W. Schnell and B. Zotter, CERN report ISR-GS-RF/76-26 (1976).

5. D. Mohl and H. Schonauer, Proc. 8th Int. Conf. on High Energy Accelerators, Stanford, p. 380 (1974).

6. G. Swain, to be published in Proc. Int. Workshop on Hadron Facility Technology, Santa Fe, New Mexico (1987). 